\title{
La formation pratique des enseignants : regard sur le système nigérien
}

\section{Chronique internationale}

\section{Introduction}

Pour améliorer la qualité de l'enseignement et de la formation dans les Écoles normales d'instituteurs (ENI), le ministère de l'Enseignement primaire, de l'Alphabétisation, de la Promotion des langues nationales et de l'Éducation civique (MEP/A/PLN) a révisé le programme de formation (MEBA, 2006). L'objectif est de passer d'un modèle trop théorique (ne permettant pas une exploitation professionnelle des savoirs acquis) à un modèle axé prioritairement sur l'articulation "Pratique-théorie-Pratique $(\mathrm{PTP})$ ». Cette nouvelle approche visant la professionnalisation de la fonction enseignante accorde une place de choix aux stages. Ceux-ci constituent ainsi l'ossature de la formation dans les ENI.

Les ENI sont chargées de la formation pratique des enseignants du primaire, c'est-à-dire des instituteurs et des instituteurs adjoints tandis que l'École normale supérieure (ENS) est, sous la tutelle du ministère de l'Enseignement supérieur, responsable de la formation pratique des futurs enseignants du secondaire (futurs enseignants du collège et du lycée) et des futurs formateurs de futurs enseignants (futurs conseillers pédagogiques et inspecteurs). 


\section{La formation pratique des enseignants au primaire (MEN, 2008)}

\subsection{Principes de la formation}

Pour partir de la pratique, la formation des futurs enseignants les outille à une interrogation permanente sur leurs pratiques de classes afin de les amener à une pratique réfléchie.

Les résultats aux tests d'entrée dans les ENI des futurs enseignants révèlent que la qualité de la langue d'enseignement laisse à désirer. Pour remédier à cette insuffisance, le MEP/A/PLN a mis un accent particulier sur la maitrise du français dans la formation initiale des futurs enseignants.

Pour préparer les futurs enseignants à affronter les difficultés pratiques du métier, la formation encourage l'autoformation et la formation permanente à travers les compétences liées à la recherche, au traitement ou à l'utilisation de l'information et à la résolution de problèmes.

La formation à l'évaluation à l'ENI prépare les futurs enseignants dans trois domaines : évaluer les prérequis des élèves avant d'aborder de nouveaux apprentissages (fonction d'orientation); évaluer les élèves en cours d'apprentissage (fonction de régulation); établir un bilan et déterminer si l'élève a réalisé les acquisitions nécessaires pour aborder des apprentissages de niveau supérieur ou obtenir une certification (fonction de certification).

\subsection{Orientations pédagogiques du programme}

Les programmes d'études des ENI reposent sur le paradigme socioconstructiviste qui réfère à la construction des connaissances par l'apprenant en situation dans un contexte social déterminé.

Le profil de sortie décrit les compétences, activités, tâches et habiletés que le formé est en droit d'acquérir à la fin de sa formation professionnelle. Le programme d'études s'appuie sur neuf compétences relevant de trois grands domaines représentant le champ d'activités de l'enseignant : compétences en enseignement; compétences en administration et en gestion et compétences en maitrise des contenus à enseigner au primaire.

\subsection{Organisation de la formation}

La démarche privilégiée dans la formation initiale des enseignants se fonde sur l'enseignement de préalables théoriques et sur l'apport de modèles (leçons modèles, plans de leçons) conçus pour être appliqués en stages pratiques et en situation de classe. Cette démarche s'est avérée inefficiente, car elle entérine la rupture entre la théorie et la pratique et aboutit à l'incapacité des futurs enseignants de synchroniser en situation réelle les préalables théoriques et les modèles enseignés.

Les différents réaménagements des programmes de formation initiale des enseignants ont surtout consisté à augmenter le nombre de disciplines enseignées ou la durée des stages pratiques. Or, cela ne peut suffire pour atteindre les objectifs assignés à la formation. 
La nouvelle démarche vise à intégrer la formation professionnelle des enseignants dans la réalité du milieu afin de les doter de compétences permettant d'affronter efficacement la réalité scolaire en changement perpétuel.

Conformément aux dispositions du programme révisé des ENI, la formation est organisée en unités de formation (UF) suivant le modèle PTP. Celles-ci constituent la base du système de formation initiale des enseignants. Elles définissent les compétences à développer suivant les différentes étapes de la scolarité primaire :

- $\quad$ les compétences de base en lecture, écriture et mathématiques sont développées en UF1 (CI$\mathrm{CP})$;

- $\quad$ l'UF2 (CE1-CE2) ou l'enseignement élémentaire prépare à la lecture courante, à la langue écrite, aux mathématiques et à l'utilisation des notions spatio-temporelles. Elle prépare aussi à l'initiation des élèves aux activités pratiques, productives et à la technologie;

- $\quad$ l'UF3 (CM1-CM2) ou l'enseignement moyen consolide et développe les compétences des sous-cycles précédents.

Il s'agit de définir les compétences à développer chez les futurs enseignants pour ce qui est de chaque sous-cycle dans la triple perspective de la connaissance de l'enfant, des matières à enseigner et de la maitrise des méthodes pour enseigner et évaluer.

Les UF fusionnent les enseignements de psychopédagogie et de pédagogie spéciale et pratique. Les enseignants de ces deux disciplines organisent les activités des UF autour des compétences suivantes : a) planifier les activités d'enseignement, d'apprentissage et d'évaluation dans les différentes disciplines par niveau et par UF; b) construire des séquences d'apprentissage; c) mettre en ouvre et gérer les apprentissages par niveau et par UF; d) évaluer les acquis des élèves et proposer des stratégies de remédiation.

Ces activités proposent aux futurs enseignants de travailler individuellement ou en groupe sous la supervision des deux formateurs. Chaque UF débute par un stage d'observation et finit par un stage pratique. Des séances de simulation de pratique de classe sont organisées en microenseignement ou dans les écoles annexes pendant cette période. Les enseignements dans les autres disciplines se poursuivent normalement au cours des UF.

\subsubsection{Stages de sensibilisation}

Les futurs enseignants observent et analysent, pendant une semaine en début d'année avant le début des enseignements, l'organisation et le fonctionnement de la rentrée scolaire, de l'école et des classes.

\subsubsection{Apprentissages de base}

Ils assurent l'acquisition des compétences professionnelles et celles liées à la gestion et à la maitrise des contenus enseignés à l'école primaire. Ils se déroulent, en début d'année scolaire, pendant neuf semaines pour le cycle « instituteur » et 24 semaines pour le cycle « instituteur adjoint ». Durant cette période, les futurs enseignants effectuent des séances d'observation dans les écoles annexes ou des séquences de démonstration dans les salles de microenseignement. 
Les apprentissages de base sont sanctionnés par des évaluations :

- $\quad$ en ce qui touche les compétences du domaine de l'enseignement, des contrôles continus sont organisés en psychopédagogie et en pédagogie spéciale et pratique, une évaluation sommative intervient à la fin de l'étude de chaque compétence;

- l'évaluation des compétences en administration, en gestion et en maitrise des contenus enseignés au primaire intervient sous forme de contrôles continus disciplinaires. L'évaluation de fin d'études porte sur la maitrise des compétences.

\subsubsection{Stages d'observation}

Avant les unités de formation, les objectifs sont précisés et une grille d'observation est élaborée pour guider les observations. Ces stages permettent ainsi aux futurs enseignants d'observer, pendant une semaine, la conduite des enseignements en classe des enseignants. Les observations des futurs enseignants sont analysées à leur retour à l'ENI.

\subsubsection{Stages pratiques guidés}

À la fin de chaque UF, ces stages permettent aux futurs enseignants de s'exercer, pendant deux semaines, à la conduite de classe sous la supervision de l'enseignant titulaire et des formateurs de l'ENI (inspecteurs). Les enseignants de psychopédagogie et de pédagogie spéciale et pratique sont responsables de l'organisation et de la gestion de ces stages.

Le stage pratique guidé est évalué. Chaque futur enseignant obtient au moins une note de stage dans chaque sous-cycle. La note de stage est la moyenne des notes attribuées par l'enseignant titulaire de classe, le formateur, l'inspecteur ou le chef du secteur pédagogique.

\subsubsection{Renforcement des capacités}

À la fin de chaque stage pratique, les enseignants remédient, pendant une semaine, aux difficultés observées durant les stages. Lors de cette période de remédiation, les autres enseignements se déroulent normalement.

\subsubsection{Stages en responsabilité}

Lors du stage en responsabilité, le futur enseignant s'initie à l'enseignement à tous les niveaux du cycle de base 1 et dans les différentes classes : multigrades, cours uniques, double flux, etc. Il est responsable de la préparation et de l'exécution de l'ensemble des activités d'enseignement sous la supervision du directeur d'école et de l'enseignant titulaire de la classe. Le stage en responsabilité est de six semaines pour le cycle instituteur et de 10 semaines pour les instituteurs adjoints. La note obtenue à ce stage est prise en compte lors de l'examen de fin de formation. Chaque futur enseignant a une note de stage en responsabilité. Cette note est la moyenne des notes attribuées par les enseignants titulaires des classes des trois niveaux et de celle attribuée par les formateurs de l'ENI ou le responsable pédagogique du secteur.

\subsubsection{Suivi et exploitation des stages}

L'école et l'ENI constituent les lieux de suivi des différents stages. À l'école, l'enseignant titulaire de la classe et le directeur jouent le rôle de tuteurs et, à l'ENI, les stagiaires sont suivis par les formateurs. 
Les inspecteurs et conseillers pédagogiques des écoles d'accueil sont impliqués dans l'encadrement des stages, en particulier des stages en responsabilité. Tous les formateurs de l'ENI participent à la conception, à la mise en œuvre et à l'exploitation des stages. Des séances d'analyses de pratiques sont organisées à la fin de chaque journée de stage entre les futurs enseignants, l'enseignant titulaire de la classe et éventuellement les formateurs.

Les diagrammes de composition et d'enchainement synthétisent l'organisation de la formation pratique des enseignants du primaire.

Instituteur

\begin{tabular}{ccccccccccccccccc}
\hline SS & AB & SO & UF1 & SP & RC & SO & UF2 & SP & RC & SO & UF3 & SP & RC & SR & RC & EF \\
\hline $1 S$ & $9 S$ & $1 S$ & $2 S$ & $1 S$ & $1 S$ & $1 S$ & $2 S$ & $2 S$ & $1 S$ & $1 S$ & $2 S$ & $2 S$ & $1 S$ & $6 S$ & $1 S$ & $1 S$ \\
\hline
\end{tabular}

Instituteur adjoint (1 ${ }^{\text {re }}$ année)

\begin{tabular}{ccccc}
\hline SS & AB & SO & UF1 & SP \\
\hline $1 S$. & $24 S$. & $1 S$. & $3 S$. & $2 S$. \\
\hline
\end{tabular}

Instituteur adjoint ( $2^{e}$ année)

\begin{tabular}{llllllllllll}
\hline RC & SO & UF2 & SP & RC & SO & UF3 & SP & RC & SR & RC & EF \\
\hline $2 \mathrm{~S}$. & $1 \mathrm{~S}$. & $4 \mathrm{~S}$. & $2 \mathrm{~S}$. & $1 \mathrm{~S}$. & $1 \mathrm{~S}$. & $4 \mathrm{~S}$. & $2 \mathrm{~S}$. & $1 \mathrm{~S}$. & $10 \mathrm{~S}$. & $3 \mathrm{~S}$. & $1 \mathrm{~S}$. \\
\hline
\end{tabular}

\section{LÉGENDE :}

SS : Stage de sensibilisation

$\mathrm{AB}:$ Apprentissages de base

$\mathrm{RC}:$ Renforcement/Consolidation

SO : Stage d'observation

UF : Unité de formation

SP : Stage pratique guidé

SR : Stage en responsabilité

EF : Évaluation finale

S. : Semaine

\subsection{Rôles des différents acteurs}

Les principaux acteurs qui interviennent pendant les stages sont :l'ENI, le directeur de l'école d'accueil, l'enseignant d'accueil et le stagiaire. 


\subsubsection{Rôles de l'ENI}

- $\quad$ sensibiliser les stagiaires;

- $\quad$ répartir les stagiaires dans les écoles d'accueil;

- $\quad$ informer les inspecteurs de l'enseignement de base;

- $\quad$ informer les écoles d'accueil par voie hiérarchique;

- $\quad$ superviser les activités de stage;

- $\quad$ exploiter la grille d'observation.

\subsubsection{Rôles du directeur de l'école d'accueil}

- $\quad$ accueillir et répartir les stagiaires selon l'unité de formation;

- faciliter l'intégration sociale du stagiaire;

- $\quad$ remplir et renvoyer à l'ENI la feuille de présence du stagiaire à la fin du stage.

\subsubsection{Rôles de l'enseignant d'accueil}

- accueillir favorablement le stagiaire;

- $\quad$ s'entretenir avec le stagiaire avant et après l'observation;

- $\quad$ expliquer et faire respecter les dispositions du Règlement intérieur;

- $\quad$ préparer et présenter obligatoirement ses leçons et ne pas céder sa classe au stagiaire.

\subsubsection{Rôles du stagiaire}

- $\quad$ observer et exploiter les réalités socioculturelle et pédagogique;

- $\quad$ se conformer au Règlement intérieur et à tous les textes qui régissent son école d'accueil au même titre que les enseignants titulaires de classes;

- $\quad$ respecter la hiérarchie pour tout contact avec l'ENI;

- $\quad$ collecter éventuellement des informations dans le cadre de la préparation de son mémoire de fin de cycle;

- $\quad$ remplir et rapporter à l'ENI la grille d'observation du stage.

\section{5. Évaluation de la formation}

\subsection{1 Évaluation du cycle des instituteurs}

L'évaluation de la formation des instituteurs se fait sous forme de contrôles continus. À la fin de la formation, la certification prend en compte les notes obtenues au cours de la formation (cours théoriques et stages pratiques) et les notes de l'examen de fin de cycle. Les notes obtenues au cours de la formation comptent pour $2 / 3$ et la note de l'examen pour $1 / 3$.

\subsection{2 Évaluation du cycle des instituteurs adjoints}

Pour les élèves de première année, deux évaluations sont prévues en janvier et en mai. Elles consistent en une sommation de l'ensemble des notes des contrôles continus organisés pour ce qui est des disciplines et de la note du stage pratique. La moyenne des deux évaluations détermine le passage en deuxième année. Un arrêté ministériel précise les critères de promotion, de redoublement et d'exclusion. 
Pour la deuxième année, l'évaluation se fait essentiellement sous forme de contrôles continus et de notes de stages. La certification de la formation prend en compte la moyenne des notes des contrôles continus et des stages pratiques et la moyenne des notes obtenues à l'examen de fin de formation (y compris la note du mémoire). Les notes obtenues au cours de l'année scolaire comptent pour 2/3 et la note de l'examen pour 1/3. Le tableau 1 présente les coefficients par discipline.

Tableau 1

Coefficients des disciplines.

\begin{tabular}{lc}
\hline Disciplines & Coefficients \\
\hline Psychopédagogie & 3 \\
Pédagogie spéciale et pratique & 3 \\
Morale professionnelle et législation & 1 \\
Sociologie de l'éducation & 1 \\
Éducation physique et sportive & 1 \\
Français & 2 \\
Mathématiques & 2 \\
Langues nationales & 1 \\
Compétences de vie & 1 \\
Mémoire & 2 \\
Stage pratique & 3 \\
\hline
\end{tabular}

De ce qui précède, il apparait que la force majeure des ENI réside dans le fait qu'elles disposent d'un guide de stages. Ce guide permet aux enseignants des établissements d'accueil, aux formateurs de terrain (inspecteurs de l'enseignement primaire et conseillers pédagogiques) et aux formateurs des ENI de participer, tant bien que mal, à la formation des futurs enseignants. Cependant, la gestion des stages est une gageure pour les ENI, les acteurs de terrain et les stagiaires. Les difficultés dans la conduite des stages des futurs enseignants sont principalement liées à la méconnaissance ou à l'incapacité des différents acteurs à s'adapter à leurs rôles.

\section{La formation pratique des enseignants au secondaire}

L'ENS ne dispose pas d'un guide de stage. Elle navigue à vue et privilégie des stages disciplinaires, ce qui condamne les futurs enseignants du secondaire (futurs enseignants du collège et du lycée) et les futurs formateurs de futurs enseignants (futurs conseillers pédagogiques et inspecteurs) à réaliser un apprentissage partiel. Cette approche (évaluation) sommaire des stages affecte de manière significative le caractère global de l'apprentissage des stages. En effet, les activités en milieu réel, celles auxquelles le stagiaire va être confronté pendant le stage, sont essentiellement globales : multiplicité des variables appartenant à différents domaines, organisation dans la durée, etc. À la différence de l'aspect disciplinaire de l'enseignement, l'expérience de stage n'est ni organisée ni vécue par matières. Ainsi, l'approche disciplinaire se montre insuffisante, car elle ne permet pas de prendre en compte 
l'expérience de stage dans sa globalité. Le retour de stage doit donc prendre la forme d'une session au cours de laquelle alterneront des entretiens individuels entre les formateurs et les stagiaires, des séances de travail entre stagiaires et des séances collectives réunissant le groupe de stagiaires et l'équipe de formation, toutes disciplines confondues (Pelpel, 1989).

Dans ces conditions, à l'ENS les stages s'inscrivant dans une perspective d'évaluation, le stagiaire à tout intérêt à se conformer à la norme. Dans ce cas, le stage ne constitue pas véritablement un moment de formation, mais un simple rituel auquel il faut se soumettre pour obtenir tel diplôme ou telle attestation.

Le temps n'est-il pas venu de s'interroger sur les conditions de réussite de l'apprentissage global?

\section{Références}

Ministère de l'Éducation de base et de l'Alphabétisation (MEBA). (2006). Politique nationale de formation initiale et continue des enseignants de l'éducation de base. Niamey: Agence canadienne de développement international.

Ministère de l'Éducation nationale (MEN). (2008). Programmes d'études des écoles normales d'instituteurs. Niamey : MEN.

Pelpel, P. (1989). Les stages de formation. Paris : Bordas. 